\title{
Distal regulation of alternative splicing by splicing enhancer in equine $\beta$-casein intron 1
}

\author{
TINA LENASI, ${ }^{1,2}$ B. MATIJA PETERLIN, ${ }^{2}$ and PETER DOVC ${ }^{1}$ \\ ${ }^{1}$ University of Ljubljana, Biotechnical Faculty, Department of Animal Science, Groblje 3, SI-1230 Domzale, Slovenia \\ ${ }^{2}$ Departments of Medicine, Microbiology and Immunology, Rosalind Russell Medical Research Center, University of California \\ at San Francisco, San Francisco, California 94143, USA
}

\begin{abstract}
The complexity of cotranscriptional splicing is reflected in the coordinated interplay between various cis-elements and transacting factors. In this report, we demonstrated that a cis-element in intron 1 of the equine $\beta$-casein gene (intronic splicing enhancer 1, ISE1) increases the inclusion of all weak exons in its pre-mRNA. The ISE1 also functioned on a hybrid transcript, which was transcribed from the $\alpha$-globin promoter, where it increased the inclusion of the human fibronectin EDA exon and the $\beta$-casein exon 5. The region of ISE1 necessary for its function included the same sequence as is found in some exonic splicing enhancers. Since the ISE1 influenced the splicing of the entire transcript from intron 1, we propose a model for the cotranscriptional splicing of $\beta$-casein mRNA, where the $5^{\prime}$ end of the growing transcript remains associated with the $C$-terminal domain of RNA polymerase II. Thus, the ISE1 remains in close proximity to the mRNA exit groove throughout transcription and influences all weak exons as soon as they are copied.
\end{abstract}

Keywords: splicing enhancer; alternative splicing; cotranscriptional processing; weak exon; casein gene

\section{INTRODUCTION}

The processing of eukaryotic pre-mRNA is coupled with transcription (Goldstrohm et al. 2001; Howe 2002; Maniatis and Reed 2002). The major link between them is the Cterminal domain (CTD) of RNA polymerase II (RNA pol II), whose phosphorylation represents a switch for the elongation and cotranscriptional processing of primary transcripts (Weeks 1993; Hirose and Manley 2000; Zeng and Berget 2000; Wetterberg et al. 2001). Demonstrations of connections between nascent transcripts, splicing factors, and the transcriptional machinery provide reliable evidence that splicing occurs cotranscriptionally (Neugebauer and Roth 1997; Hirose and Manley 2000; Kornblihtt 2004). Indeed, the role of phosphorylated CTD in the splicing process was proven by transcription assays with truncated forms of the CTD, where splicing was inhibited considerably (McCracken et al. 1997). These data are consistent with the finding that the CTD is adjacent to the exit groove of premRNA (Cramer et al. 2001).

Reprint requests to: Tina Lenasi, Departments of Medicine, Microbiology and Immunology, Rosalind Russell Medical Research Center, University of California at San Francisco, 533 Parnassus Avenue, San Francisco, CA 94143-0703, USA; e-mail: vlenasi@itsa.ucsf.edu; fax: (415) 502-1901.

Article published online ahead of print. Article and publication date are at http://www.rnajournal.org/cgi/doi/10.1261/rna.7261206.
Alternative splicing presents additional complexity to the regulation of cotranscriptional processing of pre-mRNA (Black 2003). By producing different mRNA forms from a single gene it contributes to proteome diversity (Black 2000; Maniatis and Tasic 2002). Exons with weak splice sites (weak exons) are included alternatively in the processed mRNA. The efficiency of the inclusion of weak exons depends on splicing activators or repressors, RNA secondary structure, and promoter structure.

Cis-elements that bind splicing activators or repressors are usually located within weak exons (exonic splicing enhancers, ESEs; exonic splicing silencers, ESSs) or flanking introns (intronic splicing enhancers, ISEs; intronic splicing silencers, ISSs). Splicing activators are primarily members of the serine/arginine-rich family (SR proteins) (Graveley 2000) and often bind the purine-rich ESEs (Liu et al. 1998). It is likely that SR proteins are involved in recruiting general splicing factors to aberrant splice sites in weak exons (Caceres and Kornblihtt 2002). Thus, weak exons are under evolutionary constraint to include ESEs.

The range of the direct influence of cis-elements on the splicing of weak exons is small, on the order of one to several hundred nucleotides from the affected exon (Muro et al. 1999; Schaal and Maniatis 1999). Lately it has been shown that they can act also from a specific weak exon on another weak exon, which are separated by several kilobases (Fededa et al. 2005). In this case, their influence is indirect 
in that they recruit specific splicing activators to the upstream weak exon and these activators are somehow more accessible to the downstream exon.

In addition to the RNA sequence, RNA secondary structure also plays an important role in the splicing process. For example, the exposure of key cis-elements involved in the splicing process allows them to recruit splicing activators and thus increase the inclusion of weak exons in the mRNA. Also, the spatial modification of the distance between these elements strengthens or weakens their effect (Muro et al. 1999; Baraniak et al. 2003; Buratti and Baralle 2004; Buratti et al. 2004).

Apart from the role of splicing enhancers and silencers, promoter structure and occupation influence the intensity of the inclusion of weak exons in processed mRNA (Cramer et al. 1997; Kadener et al. 2002; Pagani et al. 2003). Presumably, the promoter influences the recruitment of different splicing factors to RNA pol II or of factors that influence its processivity (Cramer et al. 2001; Kadener et al. 2002; de la Mata et al. 2003; Pagani et al. 2003). For the most part, these are factors that differentially phosphorylate the CTD (Komarnitsky et al. 2000; Fong and Bentley 2001; Fong and Zhou 2001; Bird et al. 2004). Thus, the intensity of the inclusion of weak exons most likely depends on the combined effects of ESEs, ISEs, ESSs, ISSs, RNA secondary structure, and promoter structure and occupation.

Extensive exon skipping was observed in the casein transcripts of various species (Johnsen et al. 1995). Therefore, casein genes are good candidates for studying the cotranscriptional splicing process. There have been two reports on alternative splicing of $\alpha_{S_{1}}$-casein mRNA in horses (Milenkovic et al. 2002; Lenasi et al. 2003). In this study, we discovered a splicing enhancer in $\beta$-casein intron 1 (ISE1) that increases the inclusion of several weak exons. The distal and global functions of ISE1 elucidate the complexity of splicing mechanism in eukaryotes.

\section{RESULTS}

\section{Determination of splicing patterns in equine $\alpha_{S 1}-$ and $\beta$-casein mRNA}

To identify alternatively spliced exons in equine $\alpha_{\mathrm{S1}^{-}}$and $\beta$-casein mRNAs as well as putative differences in their splicing patterns, we analyzed RNA isolated from the milk of 30 Lipizzan mares by reverse transcriptase polymerase chain reaction (RT-PCR). To prove that all alternatively spliced products were exported from the nucleus into the cytoplasm, total and cytoplasmic RNAs were used as templates. Amplified fragments were analyzed by gel electrophoresis and sequencing and were quantified by capillary

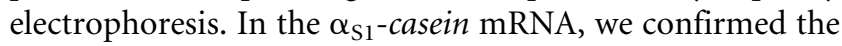
presence of two previously described alternatively included exons (Milenkovic et al. 2002; Lenasi et al. 2003), exon 8 (GenBank AY579422) and exon 15 (GenBank AY579423)
(Fig. 1, AEX8, AEX15; Fig. 2D). In $\beta$-casein mRNA, we found two alternatively included exons, exon 5 (GenBank AY579427) and exon 8 (GenBank AY579424), and an alternative donor splice site in exon 7 (Fig. 1, BEX5, BEX8 and BEX7, respectively; Fig. 2A,B,C). The use of the latter splice site resulted in mRNA forms that lacked the first $396 \mathrm{nt}$ of exon 7. The alternative inclusion of the five weak exons is most likely the consequence of nonconsensus donor and/or acceptor splice sites (Fig. 1, underlined).

To determine the inclusion of each of the two weak exons from the $\alpha_{S 1}$-casein gene, RT-PCR analyses were performed with two pairs of primers (Fig. 2D). To determine the inclusion of each of the two exons from the $\beta$-casein gene separately (Fig. 2B) and simultaneous alternative inclusion of all weak exons from the $\beta$-casein gene $(5,8$, and part of exon 7; Fig. 2C), RT-PCR analyses were performed with three pairs of primers (P1/P2 for exon 5, P3/P4 for exon 8 , and $\mathrm{P} 5 / \mathrm{P} 4$ for all of the three exons; positions of the primers are presented on Fig. 2A). All animals showed a single splicing pattern for the inclusion of the two weak exons in the $\alpha_{\mathrm{S}_{1}}$-casein mRNA (Fig. 2D). However, amplification of the $\beta$-casein mRNA (Fig. 2B,C) revealed three distinct splicing patterns for the population of 30 mares. Comparisons of these patterns showed that the inclusion of exons 5,8 , and the use of the alternative donor splice-site in exon 7 are associated. Altogether, four processed $\beta$-casein transcripts were observed because of different inclusions of the weak exons (Fig. 2A, right). The three splicing patterns

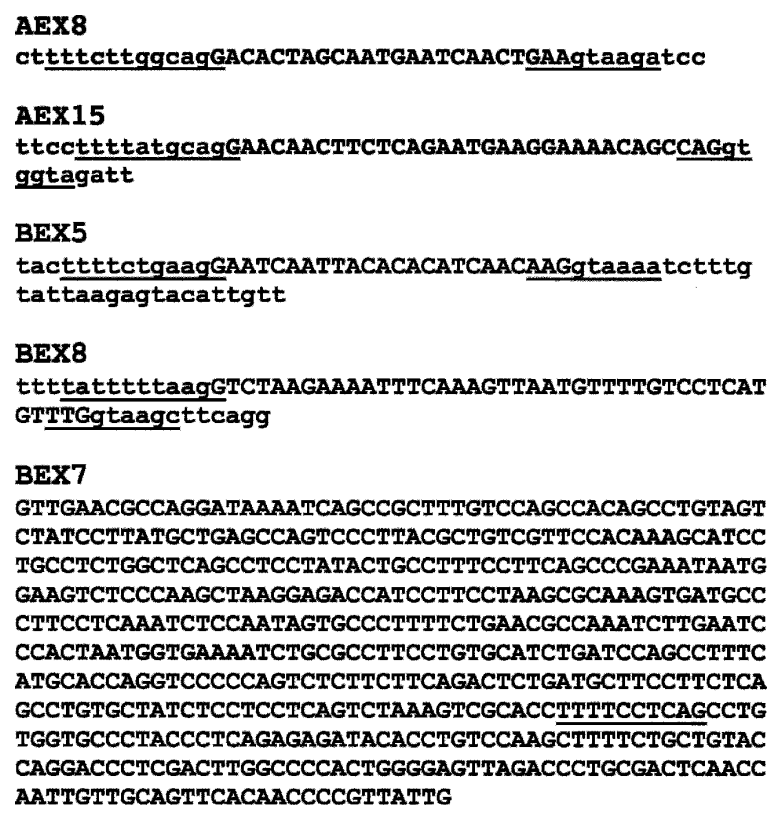

FIGURE 1. Alternatively spliced exons in equine $\alpha_{\mathrm{S1}^{-}}$and $\beta$-casein mRNA. Nucleotide sequence of the $\alpha_{S 1}$-casein exons 8 (AEX8) and 15 (AEX15) and the $\beta$-casein exons 5 (BEX5), 8 (BEX8), and 7 (BEX7). Nucleotides in exons are written in uppercase, nucleotides in introns in lowercase. Donor and acceptor splice sites are underlined; the alternative donor splice-site in exon 7 is underlined. 

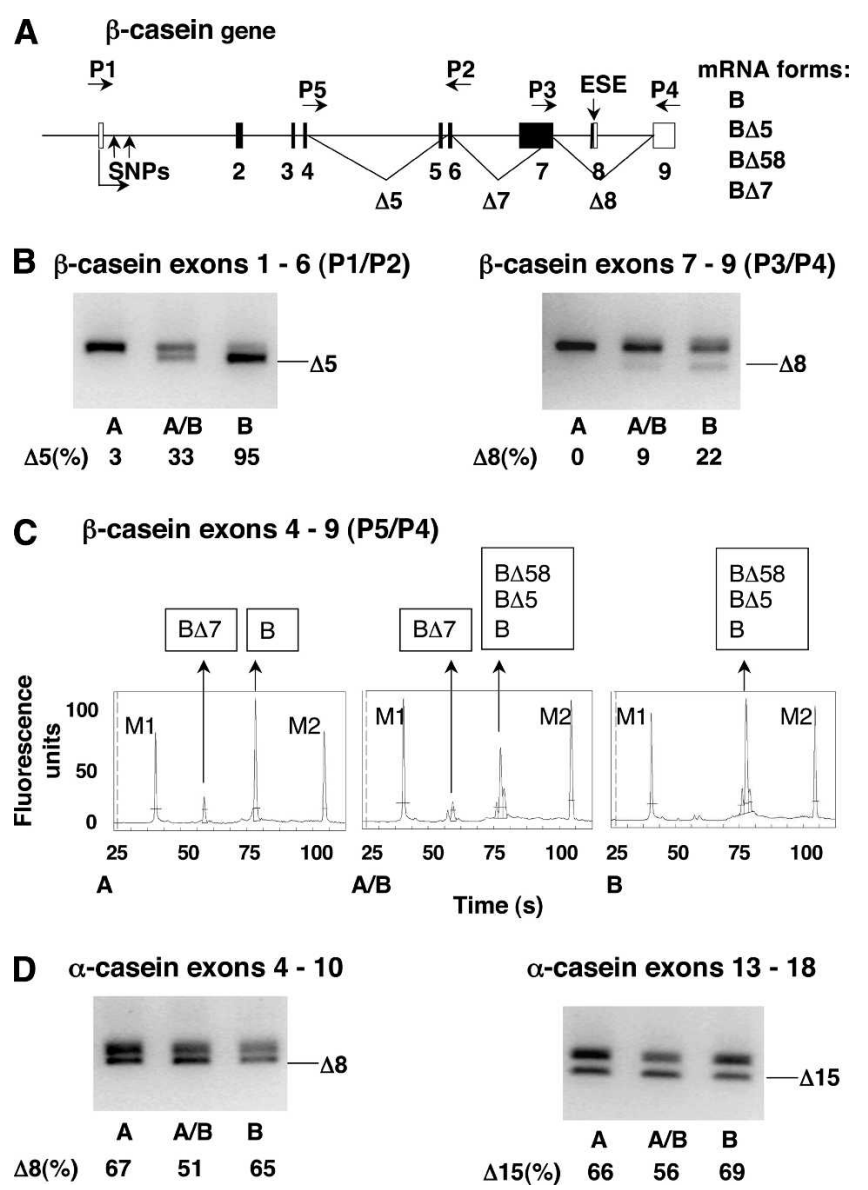

FIGURE 2. Splicing patterns of equine $\beta$ - and $\alpha_{\mathrm{S} 1}$-casein mRNA. $(A)$ Structure of $\beta$-casein gene depicted to scale; for intronic regions, the length of bovine introns is considered, and noncoding and coding exons are indicated in white and black boxes, respectively. Alternative splicing of exons 5, 8, and partially 7 is marked with lines. Alternative splicing results in four mRNA forms (right), the form with all exons is marked with $\mathrm{B}$, other forms are marked with $\mathrm{B} \Delta$, followed by numbers of skipped exons. Arrows above the gene indicate primers P1-P5. Two SNPs in intron 1 and ESE in exon 8 are indicated by arrows. $(B, C, D)$ RNA was isolated from mare's milk; splicing variants were detected by RT-PCR and analyzed by electrophoresis. A, A/B, and B are representative samples of three $\beta$-casein splicing patterns. $(B, D)$ Alternative inclusion of one weak exon at a time; analysis of fragments on $2 \%$ agarose gels. Upper fragments represent forms with included exon; lower fragments represent forms with skipped exon and are indicated with $\Delta$ followed by the exon number. The numbers below each lane are the percentage of skipping of the weak exon obtained by capillary electrophoresis of PCR products (mean value of analyzed samples). (B) Alternative inclusion of the $\beta$-casein exons 5 (left, primers $\mathrm{P} 1 / \mathrm{P} 2$ ) and 8 (right, primers $\mathrm{P} 3 / \mathrm{P} 4$ ). (D) Alternative inclusion of the $\alpha_{S 1^{-}}$ casein exons 8 (left) and 15 (right). (C) Alternative inclusion of the $\beta$ casein exons 5, 8, and partially 7 (primers $\mathrm{P} 5 / \mathrm{P} 4$ ); analysis of fragments by capillary electrophoresis. Peaks marked with M1 and M2 represent size markers (100 and 10,000 bp). Analyzed fragments representing four mRNA forms (detection limit was set above six fluorescent units) are indicated in frames above the peaks.

differed in the presence and relative quantities of the four transcripts. Thus, the samples from the 30 mares fell into one of the following three groups representing the following $\beta$-casein splicing patterns: (A) exons 5 and 8 were included in the processed mRNA and the alternative donor splice site in exon 7 was used alternatively (Fig. 2B,C, sample A); (A/B) exons 5 and 8 were alternatively included in the processed mRNA and the alternative donor splice site in exon 7 was used alternatively (Fig. 2B,C, sample A/B); and (B) exons 5 and 8 were alternatively included in the processed mRNA, with a higher ratio of skipped/included exons 5 and 8 than in the splicing pattern $\mathrm{A} / \mathrm{B}$. The alternative donor splice site in exon 7 was not used (Fig. 2B,C, sample B).

The differences in exon inclusion among the three splicring patterns were more pronounced for exon 5 . However, exon 8 was more frequently included in the processed mRNA. The reason for preferential inclusion of exon 8 probably lies in its putative ESE (AGAAAAU), which was shown to increase the inclusion of weak exons (Matter et al. 2002).

\section{Screening $\beta$-casein gene regions for sequence polymorphisms}

Based on our finding that inclusions of all weak exons from the $\beta$-casein gene are interrelated, we postulated the presence of a unique cis-element in the $\beta$-casein gene that increases the selection of all weak splice sites. To locate this element, we isolated DNA from the blood of the 21 mares and sequenced parts of the $\beta$-casein gene that could be involved in the splicing process. No polymorphisms were found in the weak exons or splice sites. Thus, we also sequenced introns flanking weak exons, the proximal promoter, the first intron, and the $3^{\prime}$ UTR.

Altogether, 13 polymorphisms were found. Eleven of these were not associated with the splicing patterns. However, two single nucleotide polymorphisms (SNPs) in intron 1 were associated with splicing patterns in the samples from all 21 mares. These two SNPs were A/T at position +119 and $\mathrm{C} / \mathrm{T}$ at position +269 in the $\beta$-casein gene. Both SNP variants were found to be linked; samples with A at position +119 always had $\mathrm{T}$ at position +269 (A-T variant), and those with $\mathrm{T}$ at position +119 always had $\mathrm{C}$ at position +269 ( $\mathrm{T}-\mathrm{C}$ variant). The samples with the A-T variant always resulted in the splicing pattern $\mathrm{A}$ and those with $\mathrm{T}-\mathrm{C}$ variant always resulted in the splicing pattern $\mathrm{B}$. Heterozygosity $(\mathrm{A} / \mathrm{T}-\mathrm{T} / \mathrm{C})$ in the two SNPs resulted in the splicing pattern $\mathrm{A} / \mathrm{B}$ (Table 1).

Although a linkage between the two SNPs and some other polymorphisms with influence on the splicing process is possible, this scenario can be excluded, because the two closest polymorphisms (200 bp upstream of the first SNP and $600 \mathrm{bp}$ downstream of the second SNP) are not linked to these two SNPs. Thus, we assumed that the SNPs in intron 1 are the reason for the observed different splicing patterns. The association of the two SNPs with the splicing patterns, together with the quantities of mRNA forms corresponding to the individual splicing patterns, are presented in Table 1. 
TABLE 1. Association of $\beta$-casein splicing patterns with the two SNPs in intron $1(100 \%$ agreement)

\begin{tabular}{|c|c|c|c|c|}
\hline $\begin{array}{l}\text { Splicing } \\
\text { Pattern }^{\mathrm{a}}\end{array}$ & $\mathrm{SNPS}^{\mathrm{b}}$ & $\begin{array}{l}\text { Number } \\
\text { of Mares }^{\mathrm{c}}\end{array}$ & Transcripts $^{\mathrm{d}}$ & $\begin{array}{c}\text { Relative } \\
\text { Quantity (\%) }\end{array}$ \\
\hline \multirow[t]{3}{*}{ A } & A-T & 8 & $\mathrm{~B} \Delta 7$ & 17 \\
\hline & & & B & 83 \\
\hline & & & $\mathrm{B} \Delta 7$ & 18 \\
\hline \multirow[t]{4}{*}{$\mathrm{A} / \mathrm{B}$} & A/T-T/C & 11 & $\mathrm{~B} \Delta 58$ & 10 \\
\hline & & & $\mathrm{B} \Delta 5$ & 49 \\
\hline & & & B & 23 \\
\hline & & & $\mathrm{B} \Delta 58$ & 15 \\
\hline \multirow[t]{2}{*}{ B } & $\mathrm{T}-\mathrm{C}$ & 2 & $\mathrm{~B} \Delta 5$ & 70 \\
\hline & & & B & 15 \\
\hline
\end{tabular}

Each of the three splicing patterns ${ }^{\mathrm{a}}$ corresponds to one of the three variants in intron $1^{\mathrm{b}}$. Of 21 samples from mares ${ }^{\mathrm{c}}$, eight revealed splicing pattern $A$ with variant $A-T$ in intron 1,11 revealed splicing pattern $\mathrm{A} / \mathrm{B}$ and a heterozygous variant in intron 1 , and two revealed splicing pattern $B$ with variant $\mathrm{T}-\mathrm{C}$ in intron $1 .{ }^{\mathrm{d}} \mathrm{mRNA}$ forms representing individual splicing pattern are marked with B followed by $\Delta$ and numbers of skipped exons; the mRNA form with all exons included is marked with B. ${ }^{\mathrm{e}}$ Quantification was performed by capillary electrophoresis of fragments obtained by PCR with primers P5 and P4 (Fig. 2C).

If transcribed into mRNA, the sequence flanking $\mathrm{A}$ at position +119 is typical for two previously described ESEs, i.e., GAAGAA (Fairbrother et al. 2002) and AGAAAAU (Matter et al. 2002). The sequence flanking $\mathrm{T}$ at position +269 is also typical for an ESE, i.e., AGAAAAU. Thus, this finding is in agreement with the existence of the splicing enhancer in intron 1 (ISE1) in the A-T variant.

\section{Testing the function of ISE1 in concert with the $\beta$-casein promoter in a hybrid minigene}

Because the ISE1 appeared to function regardless of sequences of weak exons, we sought to determine whether it also enhances the inclusion of a heterologous weak exon. We examined its effect on splicing of transcripts from a hybrid minigene. For this purpose, we used pSVEDA Tot vector with one of the alternatively spliced exons of the human fibronectin gene (EDA exon) controlled by the human $\alpha$ globin promoter (Caputi et al. 1994). First, we tested the functionality of ISE1 in its natural environment in intron 1 of the $\beta$-casein gene. By replacing the $\alpha$-globin promoter with the $\beta$-casein promoter (1082 bp), exon 1 (48 bp), and $369 \mathrm{bp}$ of intron 1 (Bi) (GenBank AY579425), we prepared two constructs (B.A-T and B.T-C) differing only in the two SNPs in intron 1 . We also used the original vector with the $\alpha$-globin promoter (pSVEDA Tot) to examine the putative differences in the splicing of transcripts from the same minigene under the control of two completely different promoters $(\alpha$-globin and $\beta$-casein promoter $+\mathrm{Bi})$. We examined the subsequent splicing patterns in two different cell lines, 293T and HeLa cells. In both cell lines, the mRNA form with the included EDA exon was predominant from minigenes with the $\beta$-casein promoter. Only slight differences in the ratios between quantities of long versus short mRNA forms were observed in the two cell lines.

To investigate further the putative influence of ISE1, we mutated the ESE in the EDA exon (element A: GAAGAA GA $\rightarrow$ GATGCAGA) in the original construct with the $\alpha$ globin promoter, as well as in the two constructs with the $\beta$ casein promoter. This element was shown to increase drastically the inclusion of the EDA exon (Caputi et al. 1994; Muro et al. 1999; Buratti et al. 2004). Schematic presentation of the three minigenes with mutated element $A$ in the EDA exon is presented in Figure 3A (minigene with the $\alpha$ globin promoter, $\mathrm{mA}$; minigenes with the $\beta$-casein promoter differing in the two SNPs, mB.T-C and mB.A-T). We transfected $293 \mathrm{~T}$ and HeLa cells with all of the mutated constructs and analyzed transcripts from the minigenes by RT-PCR with the primers pSV5' $j$ and pSV3'j (Fig. 3B,C).

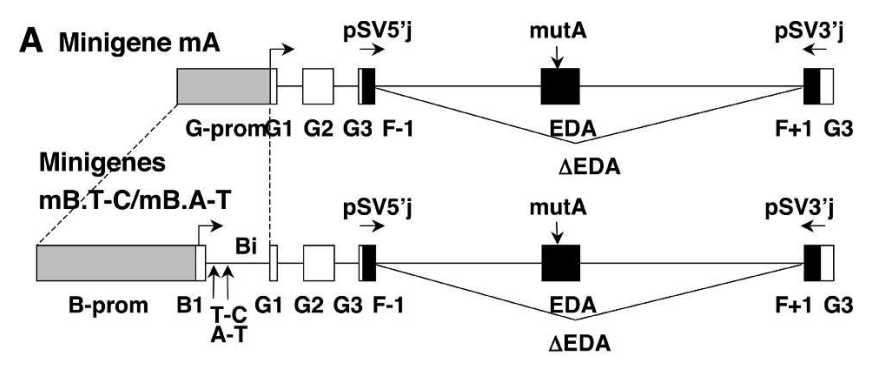

B 293T cell line

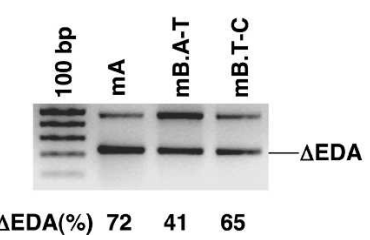

C Hela cell line

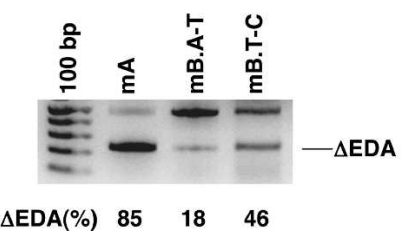

FIGURE 3. Effect of the ISE1 in $\beta$-casein intron 1 in concert with the $\beta$-casein promoter on alternative splicing of pre-mRNA from pSVEDA Tot minigene. (A) Partial structure of hybrid minigenes (minigenes $\mathrm{mA}, \mathrm{mB} . \mathrm{T}-\mathrm{C}$, and $\mathrm{mB} . \mathrm{A}-\mathrm{T}$ ) depicted to scale. Promoters, $\alpha$-globin $(\mathrm{G})+\beta$-casein (B) exons and fibronectin exons (F-1, EDA, $\mathrm{F}+1)$ are indicated in gray, white, and black boxes, respectively. In all constructs, ESE (element A) in the EDA exon was mutated (mutA) (vertical arrow). In the construct with the $\alpha$-globin promoter $(\mathrm{mA})$, the promoter was replaced by the $\beta$-casein promoter, exon 1 , and part of intron 1 (Bi) (dashed lines). The two $\beta$-casein minigenes (mB.T-C and $\mathrm{mB} . \mathrm{A}-\mathrm{T}$ ) differ in two SNPs marked with vertical arrows. Arrows above the minigenes indicate primers $\mathrm{pSV}^{\prime} \mathrm{j}$ and $\mathrm{pSV} 3^{\prime} \mathrm{j}$ that overlap the globin/fibronectin exon boundaries. Transcription start sites are indicated by arrows. Alternative splicing of the EDA exon is marked with lines. (B) 293T and (C) HeLa cells were transfected with the constructs. Splicing products were detected by RT-PCR with pSV5'j and $\mathrm{pSV} 3$ ' $j$ primers and analyzed on $2 \%$ agarose gel. Minigenes are indicated above the lanes. Upper fragments represent forms with the included EDA exon; lower fragments represent forms with the skipped exon and are indicated with $\triangle E D A$. The numbers below each lane are the percentage of skipping of the EDA exon obtained by capillary electrophoresis of PCR products (mean value of three independent transfection experiments and RT-PCR analyses). 
Products of RT-PCR were analyzed by gel electrophoresis and sequencing and were quantified by capillary electrophoresis.

As expected from previous reports on alternative splicing experiments with pSVEDA Tot, transcription of the minigenes resulted in two mRNA forms. The long form (501 nt) included the EDA exon, which was skipped in the short form ( $\triangle \mathrm{EDA}, 231 \mathrm{nt})$. However, quantities of the two transcripts from the three minigenes differed. Differences in mRNA quantities resulting from promoter swapping have already been reported (Cramer et al. 1997; Buratti et al. 2004). Our experiment confirmed the influence of the promoter structure on the inclusion of weak exons. Namely, more forms with the skipped exon were favored from the minigene with the $\alpha$-globin promoter than from the minigenes with the $\beta$-casein promoter. In addition, our results confirmed the enhancing effect of ISE1 on the splicing of a heterologous weak exon. The enhancing effect of ISE1 was observed in 293T (Fig. 3B, cf. lanes mB.A-T and mB.T-C), as well as in HeLa cells (Fig. 3C, cf. lanes mB.A-T and $\mathrm{mB}$.T-C). As expected, from the minigene with the ISE1 in intron 1 (mB.A-T) there are apparently more mRNA forms with the included exon than there are from the minigene without the ISE1 (mB.T-C). Differences in the quantities of mRNA forms from the same minigene in each of the two cell lines are most likely the consequence of differing protein compositions in these cells (Zhang et al. 2002; Black 2003; Ule et al. 2003).

\section{Testing the function of ISE1 from different positions in the minigene with the $\alpha$-globin promoter}

In addition, we asked whether the ISE1 also functions if separated from the $\beta$-casein promoter and intron 1 . For this reason, we amplified the putative region with the splicing enhancer in intron 1 of the $\beta$-casein gene. The fragment included the A-T variant of the SNPs flanked by nucleotides that were supposedly involved in the splicing and the region between both nucleotides (altogether $202 \mathrm{bp}$ ) (Fig. 4A, ISE1). We cloned the fragment into three different positions in pSVEDA Tot as follows: the $\alpha$-globin exon 1 , the $\alpha$-globin intron 1 , and the fibronectin intron $\mathrm{F}+1$ (Fig. $4 \mathrm{~A}$, bottom). To test whether a part of ISE1 with a sequence typical for splicing enhancer (E1, GAAGAAAAT) acts as an ESE when put into a weak exon, we cloned it into the EDA exon of pSVEDA Tot (Fig. 4A, E1). In addition to these constructs, we mutated the element $\mathrm{A}$ in the EDA exon in all four minigenes. We transfected HeLa cells with these eight minigenes and with pSVEDA Tot (original and with mutated element A) and analyzed transcripts from the minigenes as described above. The ISE1 retained its function in these minigenes and, surprisingly, it increased the inclusion of the EDA exon from all three positions in the minigene. The effect was strongest with the ISE1 in the $\alpha$ globin exon 1 (minigene G1ISE1), followed by the ISE1 in
A ISE1 30 bp-GAGATATATGAAGAAAAT-127 bp-TATGAGTTTAAGAAAAT-10 bp E1 GAAGAAAAT

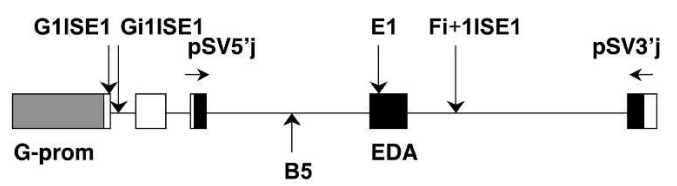

B

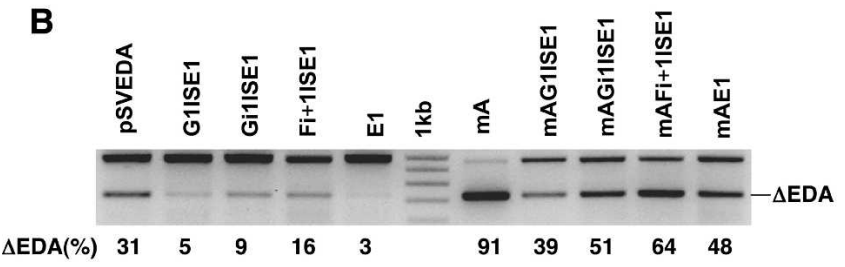

C

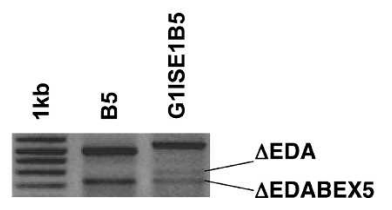

FIGURE 4. Effect of the ISE1 (202 bp of the $\beta$-casein intron 1 containing both SNPs) separated from the $\beta$-casein promoter and intron 1 on alternative splicing of pre-mRNA from pSVEDA Tot minigene. (A) The ISE1 fragment that was cloned into different positions in pSVEDA Tot and the E1 fragment that was cloned into the EDA exon (top); schematic presentation of hybrid minigene (bottom) (individual designations are explained in the legend to Fig. 3). Positions into which the ISE1 or E1 were cloned are indicated with vertical arrows and names of corresponding minigenes depicted above the arrows (e.g., cloning of the ISE1 into the $\alpha$-globin exon 1 produces plasmid G1ISE1). Cloning of the BEX5 together with parts of flanking introns into the fibronectin intron F-1 is indicated with vertical arrow and name of the minigene (B5). (B,C) HeLa cells were transfected with the constructs. Splicing products were detected as described (Fig. 3). Minigenes are indicated above the lanes. $(B)$ On the right side of the marker are minigenes with mutated element $A$ in the EDA exon (mA). Sequences and quantification of the fragments are as already described (Fig. 3). (C) The two fragments from minigene B5 (BEX5 in intron F-1) represent transcripts with the included and skipped $(\triangle \mathrm{EDA}) \mathrm{EDA}$ exon. The longest fragment from minigene G1ISE1B5 (the ISE1 in G1 exon and the BEX5 in F-1 intron) represents transcript with the included EDA and BEX5 exons. The two shorter fragments represent transcripts with the skipped EDA and included BEX5 exons ( $\triangle \mathrm{EDA})$ and with the both exons skipped ( $\triangle$ EDABEX5), respectively.

the $\alpha$-globin intron 1 (minigene GilISE1). However, it enhanced the splicing to a much lesser extent from the fibronectin intron $\mathrm{F}+1$ (minigene Fi+1ISE1) (Fig. 4B, left of the marker). The same effect was observed in transcripts from minigenes with the mutated element $\mathrm{A}$ (Fig. 4B, right of the marker). This experiment also confirmed that E1 is an ESE. When present in the EDA exon, it enhanced the inclusion of this exon to almost $100 \%$ in pSVEDA Tot vector and, to a lesser extent, in the minigene with the mutated element A (Fig. 4B, lanes E1 and mAE1, respectively). 
To prove the direct influence of ISE1 on the inclusion of weak exons from the $\beta$-casein gene, we cloned exon 5 together with parts of flanking introns (containing donor splice site, acceptor splice site, and branch point; Fig. 1, BEX5) into the fibronectin intron F-1 of pSVEDA Tot (minigene B5) and of G1ISE1 (minigene G1ISE1B5). We transfected HeLa cells with both minigenes and analyzed transcripts from the minigenes as described. The ISE1 strongly increased the inclusion of the BEX5 into processed mRNA. As expected, we observed transcripts with and without the included EDA exon. In the case of the minigene $\mathrm{B} 5$, these two forms alone were analyzed and none included the BEX5. However, among the transcripts from the minigene G1ISE1B5, the form with the included EDA exon and BEX5 prevailed. We did not detect the form with the included EDA exon and skipped BEX5. Forms with the excluded EDA exon were present in lower amounts and were composed of forms with the skipped and included BEX5 (Fig. 4C). In conclusion, the ISE1 enhances by almost $100 \%$ the inclusion of the BEX5 into the processed $\beta$-casein mRNA in mammary glands of mares, as well as in the hybrid minigene (Fig. 2B, lane A, and Fig. 4C, lane G1ISE1B5, respectively).

\section{Elements in ISE1 necessary for its function as a splicing enhancer}

To characterize more precisely elements in ISE1 that are involved in the enhancement of splicing, we created five minigenes with different parts of ISE1 deleted (Fig. 5A). All
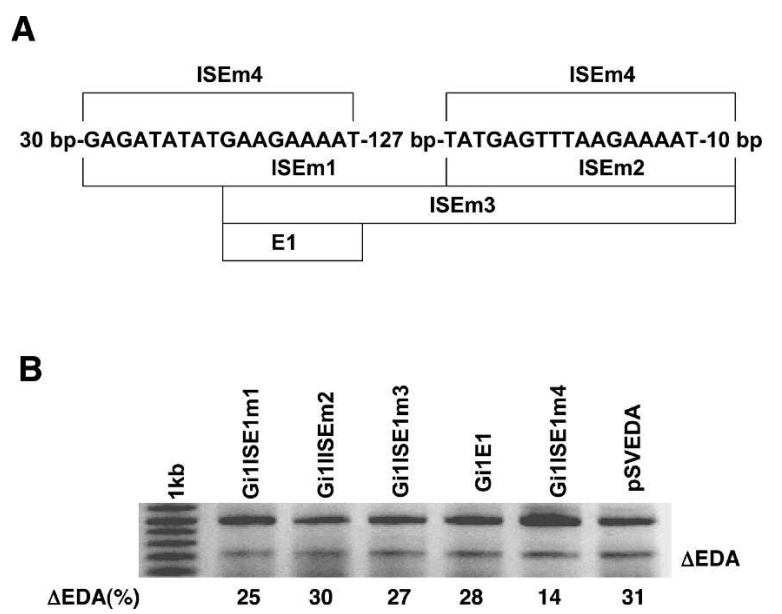

FIGURE 5. Characterization of elements in ISE1 involved in the enhancement of splicing. (A) Schematic presentation of five different deletions in the ISE1 fragment. Fragments in minigenes obtained by the deletions are presented by lines and names of mutated fragments. Deletions were performed in the minigene with the ISE1 in $\alpha$-globin intron 1 (GilISE1). (B) HeLa cells were transfected with the control plasmid pSVEDA Tot and with the five minigines indicated above the lanes (e.g., GilISE $1 \mathrm{~m} 1$ is minigene with the mutated fragment ISE1m1 in Gi1). Transcripts were analyzed for the inclusion of the EDA exon and quantification of the fragments was performed as described (Fig. 3). of the deletion mutations were performed in the minigene with the ISE1 in the $\alpha$-globin intron 1 (Gi1ISE1). HeLa cells were transfected with these five minigenes and with pSVEDA Tot as a control, and transcripts were analyzed for the inclusion of the EDA exon. The results showed that both presumed splicing enhancers (containing the two SNPs in $\beta$-casein gene) are necessary for the function of the ISE1. Namely, all minigenes without one of the two splicing enhancers had no enhancing effect on the inclusion of the EDA exon. Although E1 (GAAGAAAAT) turned out to be a strong ESE when put into the EDA exon, it was not functional in the intron 1 in the minigene (Fig. 5B, lane Gi1E1). In addition to the two proposed splicing enhancers, we observed that the first element contains additional nucleotides important for its function (GAGATATATGAA GAAAAT). Interestingly, the 127-bp-long region between both elements proved to be of minor importance for the splicing enhancer. Indeed, elements with the deletion of this region retained slightly diminished activity (Fig. 5B, lane GilISE1m4).

\section{DISCUSSION}

In this report, we demonstrated the influence of a cis-acting sequence in the equine $\beta$-casein gene on the alternative splicing of weak exons. First, the ISE1 significantly increased the inclusion of all weak exons at a distance of up to $8 \mathrm{~kb}$ in the $\beta$-casein pre-mRNA. Second, it retained its function independently of the surrounding sequence and under two different promoters ( $\beta$-casein and $\alpha$-globin) in a heterologous system, in which it enhanced the inclusion of the human fibronectin EDA exon and $\beta$-casein exon 5 . In this system, the ISE1 was functional from three different positions in the minigene. Finally, by deleting parts of the ISE1, we mapped the sequence important for its function. To recapitulate, we found a novel cis-element in intron 1 of the $\beta$-casein gene with distal, global, and promoterindependent effects on the splicing of weak exons.

Our analysis of $\beta$-casein mRNA samples revealed three distinct splicing patterns. The finding that there is a strong interconnection in the use of all weak splice sites in $\beta$-casein mRNA is astonishing. Even more surprising is that the splicing enhancer in the $\beta$-casein gene intron 1 increased the inclusion of all weak exons. Moreover, the change of just two nucleotides in the ISE1 rendered this splicing enhancer inactive. Importantly, both SNPs in the A-T variant are part of a sequence typical for two previously described ESEs, which supports the conclusion that the ISE1 increases the inclusion of weak exons. Finally, its position in the noncoding region is advantageous because there is no need for the conserved enhancer sequence to coexist with the coding sequence (Schaal and Maniatis 1999).

Our assays, using the heterologous system with the hybrid minigene confirmed the splicing-enhancing role 
of the ISE1. Surprisingly, the ISE1 did not only increase the inclusion of exons from the homologous gene ( $\beta$ casein gene) but also an exon from a heterologous gene (fibronectin gene). Furthermore, this element was also effective when inserted in the minigene with the $\alpha$-globin promoter. Thus, the ISE1 functions independently of the surrounding sequence that supports its global role. Since changing its location in the minigene did not abrogate its function, the position of the ISE1 is also not limited to an intronic sequence. Indeed, the ISE1 functioned even better when placed within the first exon. Thus, we speculate that it is favorable for the ISE1 to be closer to the $5^{\prime}$ end of the pre-mRNA. Its weaker effects from the intron immediately downstream of the weak exon support this notion.

Although splicing enhancers are usually located in weak exons or in neighboring introns, it is surprising that the ISE1 from the $\beta$-casein gene acts at a distance of several thousand nucleotides. Recently, it was shown that splicing of a certain weak exon influences splicing of another weak exon located several kilobases downstream (Fededa et al. 2005). In that study, the authors proposed a polar mechanism in which the recruitment of specific splicing activators to the upstream weak exon enhances the inclusion of the downstream weak exon by physical interactions between the two regions. Their effects were dependent on the sequence of both exons. That phenomenon is similar to our observation in that a cis-element increases the splicing of a distant weak exon. However, there are also differences between the two systems. In our study, the ISE1 acted more globally. First, the ISE1 functioned from an intron or an exon independently of the surrounding sequence, while Fededa's enhancing effect was observed only from a specific weak exon. Second, the ISE1 influenced different exons (all weak exons in the $\beta$-casein mRNA and two heterologous weak exons in the minigene), while the polar effect was exerted only on a specific downstream weak exon. Previously, the heterologous system (pSVEDA Tot vector) revealed the importance of ESEs and ISEs located in the weak exon and the importance of promoter structure and occupation in the splicing process (Caputi et al. 1994; Johnsen et al. 1995; Cramer et al. 1997; Muro et al. 1999; Buratti et al. 2004). However, the proximity of the promoter did not play a key role in the function of our ISE1. First, the ISE1 also increased the inclusion of weak exon from the position in the intron immediately downstream of the weak exon. Second, it functioned better in the minigene G1ISE1 than in the minigene GilISE1, although its position in the first minigene was only $30 \mathrm{bp}$ closer to the promoter than it was in the second one.

What is the mechanism by which the ISE 1 from the $\beta$ casein gene functions? Our results suggest that the ISE1 acts as a powerful cis-element when transcribed in the premRNA and that it remains in close proximity to unspliced introns. This cotranscriptional mechanism explains the strong and exon-independent influence of the ISE1 on the selection of all weak splice sites in the transcript. According to this model (Supplementary Figure 1), the ISE1 is functional when in close proximity to the exit groove of premRNA throughout transcription. Thus, the $5^{\prime}$ end of the growing pre-mRNA remains associated with the CTD of RNA pol II, possibly via interactions with the cap-binding complex $(\mathrm{CBC})$ and other protein(s) bound to the CTD. For the proposed mechanism, it is necessary that intron 1 is spliced out as the last intron from the pre-mRNA. Indeed, the splicing of the first intron differs from the splicing of the others in that it is enhanced by the CBC (Lewis et al. 1996). The possibility that the first intron persists in the premRNA until transcription is finished also explains why the ISE1 from exon 1 functioned better than the ISE1 from intron 1 in the hybrid minigene. When in exon 1 , the ISE1 was included in the pre-mRNA. However, it could be spliced out from the mRNA when it was located in intron 1. A similar model was lately proposed by Adamson et al. (2005) and it was based on the coupling of cleavage and polyadenylation with transcription. They showed that the CTD and $\mathrm{CBC}$ greatly enhance the cleavage reaction. Most intriguing is their novel finding that the $5^{\prime}$ ends of transcripts remain associated with the template and elongation complex after cleavage. This result strongly supports our model. Our hypothesis is also supported by the findings that the $\mathrm{CBC}$ is involved in the splicing process, that it enhances the cleavage reaction significantly, and that it cooperates with the poly(A) tail (Flaherty et al. 1997; Lewis and Izaurralde 1997; Michel et al. 2000). Indeed, all of these interactions could be executed with the $\mathrm{CBC}$ in the proximity to the transcription complex.

To summarize, our study revealed that a splicing enhancer can increase the inclusion of several weak exons in premRNA. The most important and novel finding is that this enhancer functions globally and independently of the surrounding sequence. This interesting insight into the cotranscriptional processing emphasizes that splicing activities have to be considered in the context of the entire gene and the elongation complex and not only of a single exon with adjacent elements. Nevertheless, further experiments are needed to clarify the proposed model and to elucidate the course of events that govern the cotranscriptional splicing of pre-mRNA.

\section{MATERIALS AND METHODS}

\section{RNA isolation from milk and RT-PCR}

Thirty- to $50-\mathrm{mL}$ samples of milk were taken from 30 Lipizzan mares, which were milked twice during lactation. Fifteen of the 30 mares were milked again after $1 \mathrm{yr}$, during the next lactation. Mammary gland epithelial cells were sedimented by $10 \mathrm{~min}$ centrifugation of the milk samples at $3000 \mathrm{~g}$ and $4^{\circ} \mathrm{C}$. Total RNA was isolated from the cell pellet with TRIzol reagent (Sigma) 
following the manufacturer's instructions. Cytoplasmic RNA was isolated from the cell pellet using the protocol from Gary Silverman's laboratory (http://web1.tch.harvard.edu/silverman/protocols/ nucleic/RNA/cyto.pdf).

Total and cytoplasmic RNA were used for RT-PCR reaction. cDNA was synthesized with MuLV reverse transcriptase (Perkin Elmer Applied Biosystems). For each $20 \mu \mathrm{L}$ reaction, $5 \mu \mathrm{g}$ of RNA and $0.1 \mu \mathrm{g}$ of oligo $(\mathrm{dT})_{16}$ primer were used. cDNA was then analyzed by PCR using different pairs of primers:

for $\alpha_{\mathrm{S}_{1}}$-casein exon 8: A1EX4-F 5'-TCCTCAAAGAAAGAAAGTT TCCC- $3^{\prime}$ and A1EX10-R 5'-TCGCTTGATGAAGTGCTGCT-3'; for $\alpha_{S 1}$-casein gene exon 15: A1EX13-F 5'-GCAAATACAACCAA CTCCAGC-3' and A1EX18-R 5'-GCTGGTAGGATGGTTGGAA-3'; for $\beta$-casein gene exon 5: P1 (BEX1-F) 5'-CATCCACTTACCTAC TCCTTC-3' and P2 (BEX6-R) 5'-CTCTCTTTGCTGCTGTCC CTC-3';

for $\beta$-casein gene exon 8: P3 (BEX7-F) 5'-CCTGTCCAAGCTTTT CTGCT- $3^{\prime}$ and P4 (BEX9-R) 5'-CAGATTGGAAAGATGCCAC A- $3^{\prime}$; and

for detecting all $\beta$-casein alternative mRNA forms: P5 (BEX4-F) $5^{\prime}$ AAAGCCTTTCAAGCAATGA-3' and P4 (BEX9-R).

Each PCR reaction mix $(20 \mu \mathrm{L})$ contained $50 \mathrm{ng}$ of template cDNA, $0.5 \mu \mathrm{M}$ of each primer, $0.1 \mathrm{mM}$ dNTP, $10 \mathrm{mM}$ Tris- $\mathrm{HCl}$, $50 \mathrm{mM} \mathrm{KCl}, 1.5 \mathrm{mM} \mathrm{MgCl}_{2}$, and $0.5 \mathrm{U}$ of Taq polymerase (Perkin Elmer Applied Biosystems). PCR started with initial denaturation at $94^{\circ} \mathrm{C}$ for $5 \mathrm{~min}$ and was cycled 30 times $\left(1 \mathrm{~min}\right.$ at $94^{\circ} \mathrm{C}, 45 \mathrm{sec}$ at $57^{\circ} \mathrm{C}, 30 \mathrm{sec}$ at $72^{\circ} \mathrm{C}$ ) followed by a 5 min elongation at $72^{\circ} \mathrm{C}$. PCR products were fractionated on $2 \%$ agarose gels, gel-purified, and sequenced using Big Dye Primer Cycle Sequencing Ready Reaction Kit (Perkin Elmer Applied Biosystems) in ABI Prism 310 Genetic Analyzer (Perkin Elmer Applied Biosystems). Quantification of PCR products was performed by capillary electrophoresis in the Agilent 2100 Bioanalyzer (Agilent Technologies) following the manufacturer's instructions.

\section{Determination of polymorphisms}

Nucleotide sequences of investigated regions in $\beta$-casein genes were determined by sequencing BAC clone 577C9 (pBeloBAC11, equine DNA, Laboratorie de Genetique Biochimique et Cytogenetique, Jouy-en-Josas, France). Genomic DNA was isolated from the blood of 21 Lipizzan mares (of 30 mares for which splicing patterns were examined) according to Goldfarb (1981). Polymorphisms were detected by sequencing the fragments amplified in the promoter, intron 1, introns flanking the weak exons, and $3^{\prime}$ untranslated region (UTR) of equine $\beta$-casein gene. For the amplification of the region with two SNPs in intron 1, the primers BEX1-F and BIN1-R 5'-CCCATAGTTATTCATATTGCCAAA-3' were used. Composition of the PCR mixture was the same as described previously. PCR started with initial denaturation at $94^{\circ} \mathrm{C}$ for $5 \mathrm{~min}$ and cycled 30 times $\left(1 \mathrm{~min}\right.$ at $94^{\circ} \mathrm{C}$, $45 \mathrm{sec}$ at $58^{\circ} \mathrm{C}, 40 \mathrm{sec}$ at $72^{\circ} \mathrm{C}$ ) followed by $5 \mathrm{~min}$ elongation at $72^{\circ} \mathrm{C}$. PCR products were analyzed on $2 \%$ agarose gel, gel-purified, and sequenced. All sequencing reactions were carried out using Big Dye Primer Cycle Sequencing Ready Reaction Kit (Perkin Elmer Applied Biosystems) in ABI Prism 310 Genetic Analyzer (Perkin Elmer Applied Biosystems).

\section{Construction of minigenes by cloning into pSVEDA Tot}

Two constructs (B.T-C and B.A-T) differing in the two SNPs in $\beta$ casein intron 1 were prepared by cloning the $\beta$-casein gene promoter, together with exon 1 and part of intron 1 (Bi), into pSVEDA Tot vector. The two cloned $\beta$-casein gene fragments that included $1082 \mathrm{bp}$ of promoter and $428 \mathrm{bp}$ of intron 1 (together with exon 1), and that differed in the two SNPs in intron 1 , were prepared by PCR. Primers contained ScaI (BScaI-F $5^{\prime}$-CAC CAGTACTACCCTGCCCATACTCAACAC-3') and BssHII (5'-AT GCGCGCCCATAGTTATTCATATTGCCAAA- $3^{\prime}$ ) restriction sites. Composition of the PCR mixture was the same as described previously. PCR started with initial denaturation at $94^{\circ} \mathrm{C}$ for $5 \mathrm{~min}$ and cycled 30 times $\left(1 \mathrm{~min}\right.$ at $94^{\circ} \mathrm{C}, 1 \mathrm{~min}$ at $60^{\circ} \mathrm{C}, 2 \mathrm{~min}$ at $72^{\circ} \mathrm{C}$ ) followed by $5 \mathrm{~min}$ elongation at $72^{\circ} \mathrm{C}$. Fragments were ligated with a 7150-bp ScaI/BssHII fragment of pSVEDA Tot. Ligation was performed with T4 DNA ligase (Promega), following the manufacturer's instructions. The reaction contained 30-70 ng of promoter fragment and $50 \mathrm{ng}$ of linearized pSVEDA Tot vector (Caputi et al. 1994).

\section{Construction of minigenes by mutations/insertions/ deletions into pSVEDA Tot}

Exonic splicing enhancer $\mathrm{A}$ in the human fibronectin EDA exon was disrupted by mutating two nucleotides (GAAGAAG $\mathrm{A} \rightarrow$ GATGCAGA) in the constructs (minigenes $\mathrm{mA}, \mathrm{mB} . \mathrm{T}-\mathrm{C}$, and $\mathrm{mB} . \mathrm{A}-\mathrm{T}$ ) with mutagenesis.

Splicing enhancer (E1, GAAGAAAAT) was inserted into the EDA exon in minigenes pSVEDA and $\mathrm{mA}$ (minigenes $\mathrm{E} 1$ and mAE1) with mutagenesis.

Minigenes G1ISE1, Gi1ISE1, and Fi+1ISE1 were created by inserting a 202-bp-long fragment (ISE1) from the $\beta$-casein intron 1 (from +77 to +279 ) with mutagenesis into the $\alpha$-globin exon 1 , $\alpha$-globin intron 1 , and fibronectin intron +1 of pSVEDA Tot, respectively. Minigenes mAG1ISE1, mAGi1ISE1, and mAFi+1ISE1 were created by inserting the same ISE1 fragment with mutagenesis into the $\alpha$-globin exon $1, \alpha$-globin intron 1 , and fibronectin intron +1 of minigene $\mathrm{mA}$, respectively. The fragment used for mutagenesis was PCR amplified from BAC clone 577C9.

Minigenes B5 and G1ISE1B5 were created by insertion of a 170bp-long fragment containing the $\beta$-casein exon 5 with parts of flanking introns with mutagenesis into the fibronectin intron F-1 in pSVEDA Tot and in G1ISE1, respectively. The fragment used for mutagenesis was PCR amplified from BAC clone 577C9.

Primers used for amplification of the fragments and for mutagenesis can be obtained by request.

Minigenes Gi1ISE1m1, Gi1ISE1m2, Gi1ISE1m3 and Gi1ISE1m4 were created by deletions of designated regions (Fig. 5A) in the ISE1 in minigene GilISE1 with mutagenesis. Minigine E1 was created by insertion of sequence GAAGAAAAT into the $\alpha$-globin exon 1 of pSVEDA Tot with mutagenesis. Primers used for mutagenesis can be obtained by request.

Mutagenesis reactions were performed using QuikChange XL Site-Directed Mutagenesis Kit (Stratagene).

\section{Transfections and alternative splicing assay}

Transfection was repeated three times; each transfection experiment was done in duplicate. Approximately $5 \times 10^{5}$ cells 
(HeLa or 293T) were plated on $60-\mathrm{mm}$ tissue culture dishes in DMEM growth medium (Sigma) supplemented with $10 \%$ fetal calf serum (FCS). After $24 \mathrm{~h}$, transfection with $3 \mu \mathrm{g}$ of plasmid DNA was performed using FuGENE 6 Transfection Reagent (Roche). Conditions for transfection were the same for the constructs with the $\beta$-casein promoter as for the constructs with the $\alpha$-globin promoter. After $48 \mathrm{~h}$ incubation at $37^{\circ} \mathrm{C}$ and $5 \% \mathrm{CO}_{2}$, medium was removed and each dish was covered with $2 \mathrm{~mL}$ TRIzol reagent (Sigma). Total RNA was isolated from the cells following the manufacturer's instructions and used for RT-PCR reaction. cDNA was synthesized with with MuLV reverse transcriptase (Perkin Elmer Applied Biosystems). For each $20 \mu \mathrm{L}$ reaction, $5 \mu \mathrm{g}$ of RNA and $0.1 \mu \mathrm{g}$ of antisense primer pSVcDNA (5'-GGTATTTGGAGGTCAGCA-3') were used. cDNA was then analyzed by PCR using specific pSVEDA Tot primers: sense primer pSV5'j (5'CACTGCCTGCTGGTGACTCGA-3') and antisense primer pSV3'j (5'-GCGGCCAGGGGTCACGAT-3'). Composition of the PCR mixture was the same as described previously. PCR started with initial denaturation at $94^{\circ} \mathrm{C}$ for $5 \mathrm{~min}$ and cycled 30 times $\left(45 \mathrm{sec}\right.$ at $94^{\circ} \mathrm{C}, 1 \mathrm{~min}$ at $62^{\circ} \mathrm{C}, 1 \mathrm{~min}$ at $72^{\circ} \mathrm{C}$ ), followed by $5 \mathrm{~min}$ elongation at $72^{\circ} \mathrm{C}$. PCR products were fractionated on $2 \%$ agarose gels. Individual fragments were gel-purified and sequenced using Big Dye Primer Cycle Sequencing Ready Reaction Kit (Perkin Elmer Applied Biosystems) in ABI Prism 310 Genetic Analyzer (Perkin Elmer Applied Biosystems). Quantification of PCR products was performed by capillary electrophoresis in the Agilent 2100 Bioanalyzer (Agilent Technologies).

\section{SUPPLEMENTARY DATA}

Supplementary data are available at the Peterlin laboratory home page (http://www.ucsf.edu/bmplab/pdf/Supplementary figure 1.pdf).

\section{ACKNOWLEDGMENTS}

We thank F.E. Baralle and A.F. Muro who generously provided pSVEDA Tot vector, as well as J.E. Dahlberg, M. Barboric, and A. Razpet for helpful comments on the manuscript. This work was supported by the Ministry of Education, Science and Sports of the Republic of Slovenia (P4-0220 and S4-402-001/19330/2001), by COST action B20, and by a grant from NIH (PO1 AI058708 from $\mathrm{NIH)}$ to B.M.P.

Received December 6, 2004; accepted December 1, 2005.

\section{REFERENCES}

Adamson, T.E., Shutt, D.C., and Price, D.H. 2005. Functional coupling of cleavage and polyadenylation with transcription of mRNA. J. Biol. Chem. 280: 32262-32271.

Baraniak, A.P., Lasda, E.L., Wagner, E.J., and Garcia-Blanco, M.A. 2003. A stem structure in fibroblast growth factor receptor 2 transcripts mediates cell-type-specific splicing by approximating intronic control elements. Mol. Cell. Biol. 23: 9327-9337.

Bird, G., Zorio, D.A., and Bentley, D.L. 2004. RNA polymerase II carboxy-terminal domain phosphorylation is required for cotran- scriptional pre-mRNA splicing and 3 '-end formation. Mol. Cell. Biol. 24: 8963-8969.

Black, D.L. 2000. Protein diversity from alternative splicing: A challenge for bioinformatics and post-genome biology. Cell 103: 367-370.

- 2003. Mechanisms of alternative pre-messenger RNA splicing. Annu. Rev. Biochem. 72: 291-336.

Buratti, E. and Baralle, F.E. 2004. Influence of RNA secondary structure on the pre-mRNA splicing process. Mol. Cell. Biol. 24: 1050510514.

Buratti, E., Muro, A.F., Giombi, M., Gherbassi, D., Iaconcig, A., and Baralle, F.E. 2004. RNA folding affects the recruitment of SR proteins by mouse and human polypurinic enhancer elements in the fibronectin EDA exon. Mol. Cell. Biol. 24: 1387-1400.

Caceres, J.F. and Kornblihtt, A.R. 2002. Alternative splicing: Multiple control mechanisms and involvement in human disease. Trends Genet. 18: 186-193.

Caputi, M., Casari, G., Guenzi, S., Tagliabue, R., Sidoli, A., Melo, C.A., and Baralle F.E. 1994. A novel bipartite splicing enhancer modulates the differential processing of the human fibronectin EDA exon. Nucleic Acids Res. 22: 1018-1022.

Cramer, P., Pesce, C.G, Baralle, F.E., and Kornblihtt, A.R. 1997. Functional association between promoter structure and transcript alternative splicing. Proc. Natl. Acad. Sci. 94: 11456-11460.

Cramer, P., Bushnell, D.A., and Kornberg, R.D. 2001. Structural basis of transcription: RNA polymerase II at 2.8 angstrom resolution. Science 292: 1863-1876.

de la Mata, M., Alonso, C.R., Kadener, S., Fededa, J.P., Blaustein, M., Pelisch, F., Cramer, P., Bentley, D., and Kornblihtt, A.R. 2003. A slow RNA polymerase II affects alternative splicing in vivo. Mol. Cell 12: 525-532.

Fairbrother, W.G., Yeh, R.F., Sharp, P.A., and Burge, C.B. 2002. Predictive identification of exonic splicing enhancers in human genes. Science 297: 1007-1013.

Fededa, J.P., Petrillo, E., Gelfa, M.S., Neverov, A.D., Kadener, S., Nogues, G., Pelisch, F., Baralle, F.E., Muro, A.F., and Kornblihtt, A.R. 2005. A polar mechanism coordinates different regions of alternative splicing within a single gene. Mol. Cell 19: 393-404.

Flaherty, S.M., Fortes, P., Izaurralde, E., Mattaj, I.W., and Gilmartin, G.M. 1997. Participation of the nuclear cap binding complex in pre-mRNA 3' processing. Proc. Natl. Acad. Sci. 94: 11893-11898.

Fong, N. and Bentley, D.L. 2001. Capping, splicing, and 3' processing are independently stimulated by RNA polymerase II: Different functions for different segments of the CTD. Genes \& Dev. 15: 1783-1795.

Fong, Y.W. and Zhou, Q. 2001. Stimulatory effect of splicing factors on transcriptional elongation. Nature 414: 929-933.

Goldfarb, A. 1981. Changes in the promoter range of RNA polymerase resulting from bacteriophage T4-induced modification of core enzyme. Proc. Natl. Acad. Sci. 78: 3454-3458.

Goldstrohm, A.C., Greenleaf. A.L., and Garcia-Blanco, M.A. 2001. Cotranscriptional splicing of pre-messenger RNAs: Considerations for the mechanism of alternative splicing. Gene 277: 31-47.

Graveley, B.R. 2000. Sorting out the complexity of SR protein functions. RNA 6: 1197-1211.

Hirose, Y. and Manley, J.L. 2000. RNA polymerase II and the integration of nuclear events. Genes \& Dev. 14: 1415-1429.

Howe, K.J. 2002. RNA polymerase II conducts a symphony of premRNA processing activities. Biochim. Biophys. Acta 1577: 308-324.

Johnsen, L.B., Rasmussen, L.K., Petersen, T.E., and Berglund, L. 1995. Characterization of three types of human $\alpha$ sl-casein mRNA transcripts. Biochem. J. 309: 237-242.

Kadener, S., Fededa, J.P., Rosbash, M., and Kornblihtt, A.R. 2002. Regulation of alternative splicing by a transcriptional enhancer through RNA pol II elongation. Proc. Natl. Acad. Sci. 99: 81858190.

Komarnitsky, P., Cho, E.J., and Buratowski, S. 2000. Different phosphorylated forms of RNA polymerase II and associated mRNA processing factors during transcription. Genes \& Dev. 14: 2452 2460. 
Kornblihtt, A.R., de la Mata, M., Fededa, J.P., Munoz, M.J., and Nogues, G. 2004. Multiple links between transcription and splicing. $R N A$ 10: 1489-1498.

Lenasi, T., Rogelj, I., and Dovc, P. 2003. Characterization of equine cDNA sequences for $\alpha \mathrm{S} 1-, \beta$ - and $\kappa$-casein. J. Dairy. Res. 70: 29-36.

Lewis, J.D. and Izaurralde, E. 1997. The role of the cap structure in RNA processing and nuclear export. Eur. J. Biochem. 247: 461-469.

Lewis, J.D., Izaurralde, E., Jarmolowski, A., McGuigan, C., and Mattaj, I.W. 1996. A nuclear cap-binding complex facilitates association of U1 snRNP with the cap-proximal 5' splice site. Genes. \& Dev. 10: 1683-1698.

Liu, H.X., Zhang, M., and Krainer, A.R. 1998. Identification of functional exonic splicing enhancer motifs recognized by individual SR proteins. Genes \& Dev. 12: 1998-2012.

Maniatis, T. and Reed, R. 2002. An extensive network of coupling among gene expression machines. Nature 416: 499-506.

Maniatis, T. and Tasic, B. 2002. Alternative pre-mRNA splicing and proteome expansion in metazoans. Nature 418: 236-243.

Matter, N., Herrlich, P., and Konig, H. 2002. Signal-dependent regulation of splicing via phosphorylation of Sam68. Nature 420: 691695.

McCracken, S., Fong, N., Yankulov, K., Ballantyne, S., Pan, G., Greenblatt, J., Patterson, S.D., Wickens, M., and Bentley, D.L. 1997. The C-terminal domain of RNA polymerase II couples mRNA processing to transcription. Nature 385: 357-361.

Michel, Y.M., Poncet, D., Piron, M., Kean, K.M., and Borman, A.M. 2000. Cap-Poly(A) synergy in mammalian cell-free extracts. Investigation of the requirements for poly(A)-mediated stimulation of translation initiation. J. Biol. Chem. 275: 32268-32276.

Milenkovic, D., Martin, P., Guerin, G., and Leroux, C. 2002. A specific pattern of splicing for the horse $\alpha$ S1-Casein mRNA and partial genomic characterization of the relevant locus. Genet. Sel. Evol. 34: 509-519.

Muro, A.F., Caputi, M., Pariyarath, R., Pagani, F., Buratti, E., and Baralle, F.E. 1999. Regulation of fibronectin EDA exon alternative splicing: Possible role of RNA secondary structure for enhancer display. Mol. Cell. Biol. 19: 2657-2671.

Neugebauer, K.M. and Roth, M.B. 1997. Transcription units as RNA processing units. Genes. \& Dev. 11: 3279-3285.

Pagani, F., Stuani, C., Zuccato, E., Kornblihtt, A.R., and Baralle, F.E. 2003. Promoter architecture modulates CFTR exon 9 skipping. J. Biol. Chem. 278: 1511-1517.

Schaal, T.D. and Maniatis, T. 1999. Multiple distinct splicing enhancers in the protein-coding sequences of a constitutively spliced premRNA. Mol. Cell. Biol. 19: 261-273.

Ule, J., Jensen, K.B., Ruggiu, M., Mele, A., Ule, A., and Darnel, R.B. 2003. CLIP identifies Nova-regulated RNA networks in the brain. Science 302: 1212-1215.

Weeks, J.R., Hardin, S.E., Shen, J., Lee, J.M., and Greenleaf, A.L. 1993. Locus-specific variation in phosphorylation state of RNA polymerase II in vivo: Correlations with gene activity and transcript processing. Genes \& Dev. 7: 2329-2344.

Wetterberg, I., Zhao, J., Masich, S., Wieslander, L., and Skoglund, U. 2001. In situ transcription and splicing in the Balbiani ring 3 gene. EMBO J. 20: 2564-2574.

Zeng, C. and Berget, S.M. 2000. Participation of the C-terminal domain of RNA polymerase II in exon definition during premRNA splicing. Mol. Cell. Biol. 20: 8290-8301.

Zhang, W., Liu, H., Han, K., and Grabowski, P.J. 2002. Regionspecific alternative splicing in the nervous system: Implications for regulation by the RNA-binding protein NAPOR. RNA 8: 671685 . 

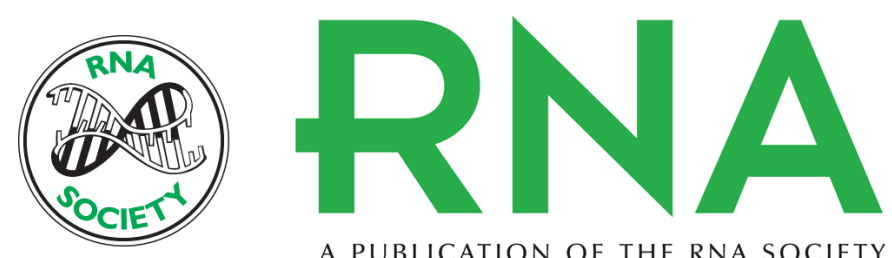

A PUBLICATION OF THE RNA SOCIETY

\section{Distal regulation of alternative splicing by splicing enhancer in equine $\beta$-casein intron 1}

TINA LENASI, B. MATIJA PETERLIN and PETER DOVC

RNA 2006 12: 498-507

References This article cites 45 articles, 28 of which can be accessed free at:

http://rnajournal.cshlp.org/content/12/3/498.full.html\#ref-list-1

\section{License}

Email Alerting Receive free email alerts when new articles cite this article - sign up in the box at the Service top right corner of the article or click here. 